\title{
Construction of genetic linkage map and identification of QTLs related to agronomic traits in maize using DNA transposon-based markers
}

\author{
Rahul Vasudeo Ramekar ${ }^{\dagger 1)}$, Kyu Jin Sa ${ }^{\dagger 1)}$, Kyong-Cheul Park ${ }^{\dagger 2}$, Neha Roy ${ }^{3)}$, Nam-Soo Kim ${ }^{3)}$ \\ and Ju Kyong Lee*1) \\ 1) Department of Applied Plant Sciences, College of Agriculture and Life Sciences, Kangwon National University, Chuncheon, 24341, \\ Korea \\ 2) Department of Agriculture and Life Industry, Kangwon National University, Chuncheon, 24341, Korea \\ 3) Department of Molecular Bioscience, Institute of Bioscience and Biotechnology, Kangwon National University, Chuncheon, 24341, \\ Korea
}

Transposable elements (TEs), are a rich source for molecular marker development as they constitute a significant fraction of the eukaryotic genome and impact the overall genome structure. Here, we utilize Mutatorbased transposon display ( $M u$-TD), and CACTA-derived sequence-characterized amplified regions (SCAR) anchored by simple sequence repeats and single nucleotide polymorphisms to locate quantitative trait loci (QTLs) linked to agriculturally important traits on a genetic map. Specifically, we studied recombinant inbred line populations derived from a cross between dent corn and waxy corn. The resulting linkage map included $259 \mathrm{Mu}$-anchored fragments, 34 SCARs, and 614 SSR markers distributed throughout the ten maize chromosomes. Linkage analysis revealed three SNP loci associated with kernel starch synthesis genes (sh2, sul, wxl) linked to either $M u$-TD loci or SSR markers, which may be useful for maize breeding programs. In addition, we used QTL analysis to determine the chromosomal location of traits related to grain yield and kernel quality. We identified 24 QTLs associated with nine traits located on nine out of ten maize chromosomes. Among these, 13 QTLs involved $M u$ loci and two involved SCARs. This study demonstrates the potential use of DNA transposon-based markers to construct linkage maps and identify QTLs linked to agronomic traits.

Key Words: genetic map, mutator, QTLs, SCAR, Zea mays.

\section{Introduction}

Maize (Zea mays ssp. mays) is among the most predominant food sources in the grass family (Poaceae), along with wheat (Triticum aestivum) and rice (Oryza sativa) (Gaut 2002). In addition, maize is an economically important crop and serves as a primary component of biofuels and animal fodder, as well as a source of byproducts used in textile, adhesive, and papermaking industries (Gaut 2002, Xu et al. 2009). Based on both endosperm and kernel constitution, maize can be classified into two categories, non-glutinous (normal maize) and glutinous (waxy maize). The distinguishing feature between normal maize and waxy maize is their relative starch content. For instance, normal maize

Communicated by Sang-Nag Ahn

Received February 15, 2018. Accepted June 14, 2018.

First Published Online in J-STAGE on August 23, 2018.

*Corresponding author (e-mail: jukyonglee@kangwon.ac.kr)

${ }^{\dagger}$ These authors contributed equally to this work starch is composed of $25 \%$ amylose and $75 \%$ amylopectin. In contrast, waxy maize starch consists exclusively of amylopectin due to a mutation or insertion in the waxy gene $(w x)$ that reduces the synthesis of amylose (Fan et al. 2008, Klosgen et al. 1986, Nelson and Rines 1962). Waxy maize is mainly cultivated and distributed in Eastern Asian countries such as China, Korea, Thailand, Vietnam, Laos, and Myanmar as a staple food and source material for industrial purposes.

In response to the shifting of diet preferences from a ricebased diet to a Western meat-based diet, waxy maize has gained popularity and is considered a valuable crop in Korea. In fact, Korea has recently increased its maize production, which will likely increase exponentially in response to future demand. Based on consumer taste and market needs, agronomic traits related to grain quality and yield are important considerations for breeders and farmers alike (Park et al. 2013, Prasanna et al. 2010).

However, improving the traits of agronomically important crops is difficult because they are complex, environmentally sensitive, and governed by different genetic components. 
For example, plant height $(\mathrm{PH})$, ear and kernel related traits, and stem thickness (ST) are factors associated with grain yield (Zhang et al. 2011). Specifically, in the waxy maize, kernel amylose content (AC) and water content (WC) improves grain texture and is favored by consumers (Ketthaisong et al. 2013, Zhang et al. 2013). To target agriculturally important traits for crop improvement, several genetic approaches have been developed. For example, past research has demonstrated that agriculturally important traits are closely associated with several quantitative trait loci (QTL) (Gupta et al. 2006). In addition to QTL mapping, genetic dissection and localization of major genes that control simple agronomic traits have been used to construct detailed genetic maps with high levels of genomic coverage and is the first step for application of molecular markers in maize breeding (Collard et al. 2005, Semagn et al. 2006). In addition, the development of molecular markers, such as single nucleotide polymorphism (SNP) and simple sequence repeats (SSR), have created the opportunity to produce dense linkage maps as well as to characterize and locate QTLs (Bernardo 2008, Grover and Sharma 2016, Prasanna et al. 2010). Indeed, with the help of molecular markers, previous studies have reported the presence of QTLs related to agronomic traits on all ten maize chromosomes (Park et al. 2013, Peng et al. 2011, Sa et al. 2015, Yang et al. 2011).

In our previous study (Sa et al. 2015), we constructed a framework map and identified QTLs for agronomic traits using 614 informative markers, including 608 SSRs and six SNPs for a RIL population derived from a cross between dent corn (Mo17) and waxy corn (KW7). However most of the SSR markers available in database no longer showed polymorphism in parental lines of RIL population limiting the chances of generating a more dense linkage map. In addition to previously developed molecular markers, mobile fragments of DNA called transposable elements (TEs) may create opportunity to produce high-density linkage map and facilitate to locate additional QTLs. TEs can play an important role in determining genomic architecture by moving to new chromosomal locations (Lisch 2013, Studer et al. 2011). In fact, $85 \%$ of the recently sequenced maize genome (B73) is comprised of multiple TE families (Schnable et al. 2009), and transposon activity has been attributed to large differences between sequenced regions of maize recombinant inbred lines (RIL) (Brunner et al. 2005, Morgante et al. 2007, Springer and Stupar 2007). Moreover, TE insertion polymorphisms in various crops have been associated with phenotypic variations in agronomically important traits (Butelli et al. 2012, Kobayashi et al. 2004, Salvi et al. 2007). In fact, the majority (66\%) of maize genes are located within $1 \mathrm{~kb}$ of an annotated transposon, which suggests that TEs play a central role in plant domestication and evolution (Schnable et al. 2009), and would thereby serve as an ideal marker system for plant breeding. Depending upon their transposition mechanism, TEs are comprised of two main groups: class I and class II DNA transposons (Wicker et al. 2007). Various TE superfamilies from both TE groups have been used to develop new markers in important crops such as rice (Branco et al. 2007, Vitte et al. 2004), maize (Du et al. 2009, Lee et al. 2005), and wheat (Queen et al. 2004, Yaakov et al. 2012). Therefore, TEs have proven to be an efficient tool for genetic mapping (Korswagen et al. 1996, Manninen et al. 2000, Rodriguez et al. 2006).

Recently, we have modified a transposon display (TD) technique to identify TEs with the Mutator $(M u)$ insertion polymorphism. $M u$ elements, a class II DNA transposon, originated in plants $60-70$ million years ago since the ancestor of maize and rice diverged (Bennetzen 1996, Robertson 1978). The $M u$ system accounts for approximately $1 \%$ of the maize genome and is considered to be one of the most complex superfamilies (Schnable et al. 2009). In maize, the $M u$ element system is highly active and has a transposition frequency of $1 \times 10^{-3}$ to $1 \times 10^{-5}$ per gene, per generation, thereby resulting in a mutation rate that is $50 \times$ that of background mutation rates (Lisch 2002, Wang et al. 2008). The high mutation rates of the $M u$ element results from its proclivity to insert into any chromosomal location (Diao and Lisch 2006). Moreover, its preference for transposing within $5^{\prime}$ untranscribed regions or exons of genes has rendered the $M u$ element an extremely popular tool for gene tagging, and cloning (Wang et al. 2008, Zhong et al. 2012).

Here we report the integration of two DNA transposonbased marker systems, $M u$-TD and Ti derived SCARs into a previously published map. We studied the chromosomal distribution of the two DNA transposon-based marker systems using anchored SSR and SNP markers. Lastly, we investigated the ability of the DNA transposon-based marker system to identify QTLs associated with important agronomic traits, including kernel water and Amylose content.

\section{Material and Methods}

\section{Plant material and DNA isolation}

We used 80 RIL populations $\left(\mathrm{F}_{7: 8}\right)$ developed from a single seed produced by a cross between Mo17 (U.S, Corn Belt maize inbred line) and KW7 (inbred line from Korean waxy maize landrace), originally developed to increase the grain yield and kernel quality of waxy maize (Sa et al. 2015). Genomic DNA was extracted from young leaf tissue approximately four weeks post-germination using a plant DNA isolation mini kit (DNeasy Plant Mini Kit, Qiagen, Hilden, Germany).

\section{Mu-TD and Ti-SCAR analysis}

The $M u$-TD protocol was performed following methods described in Ramekar et al. (2018). The specific primer sequences used are shown in Table 1. Amplification products were visualized using the gel system on a LI-COR 4300 sequencer according to the manufacturer's protocol (LI-COR Biotech, Lincoln, USA). Fluorescently-labeled DNA (50$700 \mathrm{bp} ; 50-700$ sizing standard LI-COR) served as molecular weight markers. Polymorphic parental lines were used to genotype the RIL population using 34 transposon insertion- 
Table 1. Nucleotide sequences for $B f a I$ ligation adaptors, adaptor specific primers and mutator specific primers

\begin{tabular}{|c|c|}
\hline & Ligation Adaptors \\
\hline Bfa-A1 & 5'-TAGCAAGGAGAGGACGCTGTCTGTCGAAGGTAAGGAACGGACGAGAGAAGGGAGA-3' \\
\hline \multirow[t]{2}{*}{$B f a-\mathrm{A} 2$} & 5'-TCTTCCCTTCTCGAATCGTAACCGTTCGTACGAGAATCGCTGTCTCTCCTTGC-3' \\
\hline & Adaptor specific primers \\
\hline ap1 & 5'-CGAATCGTAACCGTTCGTACGAGAATCGCT-3' \\
\hline \multirow[t]{2}{*}{ ap2 } & 5'-GTACGAGAATCGCTGTCCTC-3' \\
\hline & $M u$ specific primers \\
\hline$M u \mathrm{G} 1-1$ & 5'-GCGAAASCCYRATTTGATAATGGC-3' \\
\hline$M u \mathrm{G} 1-3$ & 5'-CCAGTGTTTGAAAATGGCGT-3' \\
\hline$M u \mathrm{G} 1-4$ & 5'-CTCAAAACAGCCTCCAAACTG-3' \\
\hline$M u \mathrm{G} 1-8$ & 5'-TCTCTAACGTGCAATCTTTCCA-3' \\
\hline$M u \mathrm{G} 2-2$ & 5'-GCGACTATTGTGTTGAAGCC-3' \\
\hline$M u \mathrm{G} 2-4$ & 5'-GGACGGGAGGATCATAATC-3' \\
\hline$M u \mathrm{G} 3-2$ & 5'-GCCTCCAATTYKTCGAAACCG-3' \\
\hline$M u \mathrm{G} 3-4$ & 5'-GCCTCCATTTCGTYGAATCCC-3' \\
\hline MuG4-1 & 5'-GCTTTGAGATTCGGAGTACCA-3' \\
\hline$M u \mathrm{G} 4-2$ & 5'-GCAAGGAACGGTGGATGTAG-3' \\
\hline$M u \mathrm{G} 4-3$ & 5'-CCGTTGGTTTGAAGATCGACGAG-3' \\
\hline$M u \mathrm{G} 4-4$ & 5'-GCAACGTTTGGAGTTTGCAG-3' \\
\hline$M u \mathrm{G} 4-6$ & 5'-TTCACGTAGCAAACGCAATC-3' \\
\hline MuG5-1 & 5'-GGGAGGGGAAAACTGATTAGAG-3' \\
\hline$M u \mathrm{G} 5-2$ & 5'-CCACTTGTAAGGTGTCGGGT-3' \\
\hline MuG6-2 & 5'-ACATTAGAGAAAGGAGGGGTTC-3' \\
\hline$M u \mathrm{G} 7-1$ & 5'-TWTGTGYATTTTGTGCACSG-3 \\
\hline$M u \mathrm{G} 7-3$ & 5'-GTCCGTGCTCACCAGATATG-3' \\
\hline$M u \mathrm{G} 7-4$ & 5'-CAAAATTGGTTGTTGCCATCGG-3' \\
\hline$M u \mathrm{G} 7-6$ & 5'-CTAATCCACATCCAGCCATTGA-3' \\
\hline
\end{tabular}

This table is modified from original article Ramekar et al. (2018).

SCARs previously developed by Roy et al. (2017). The PCR mixture $(20 \mu \mathrm{l})$ contained $20 \mathrm{ng}$ of template DNA, 10× PCR buffer, $0.2 \mathrm{mM}$ of each dNTP, $0.5 \mu \mathrm{m}$ of the forward and reverse primers, and $0.025 \mathrm{U}$ of i-Star Taq DNA polymerase (Intron Biotechnology, Korea). DNA was amplified using the following protocol: pre-denaturation at $95^{\circ} \mathrm{C}$ for $5 \mathrm{~min}$; then 20 cycles of denaturation at $95^{\circ} \mathrm{C}$ for $30 \mathrm{~s}$, then primer annealing at $56^{\circ} \mathrm{C}$ for $30 \mathrm{~s}$, extension at $72^{\circ} \mathrm{C}$ for $1 \mathrm{~min}$, and final extension at $72^{\circ} \mathrm{C}$ for $5 \mathrm{~min}$ to ensure complete extension of the PCR products. Amplicons were analyzed via gel electrophoresis with a $1.0 \%$ agarose gel. DNA fragments were visualized by ethidium bromide staining under UV light. Because both marker systems are dominant, they were manually scored as a binary response variable, with 1 (presence) and 0 (absence), respectively.

\section{Linkage map construction}

To locate $M u$-TD and SCARs on the linkage map, 614 markers (608 SSRs and 6 SNPs) previously mapped on dent $($ Mo17) $\times$ waxy corn $($ KW7) RIL populations were used as anchors (Sa et al. 2012, 2015). The nucleotide sequences of the three SNP primers (shl, sul, and $w x l)$ used in this study were reported by Lee et al. (2006). The SNP amplifications were performed by the method described by Sa et al. (2012). Markers that did not amplify $>20 \%$ in the RIL population and/or deviated from a Mendelian segregation ratio (1:1) were eliminated from the analysis. Marker integration was conducted using the inclusive composite interval mapping (ICIM) method within the QTL IciMapping software
(Meng et al. 2015). Using this software, the distance between linked markers was calculated based on a limit of detection (LOD) score of 7.0. Relative marker order within a group was determined using the algorithm nnTwoOpt, and the rippling algorithm 'COUNT' was used to fine tune the linkage map. Lastly, recombination frequencies were converted to centimorgans (cM) using Kosambi mapping functions (Kosambi 1943).

\section{Quantitative trait loci detection}

The phenotyping trait data we used for identifying agriculturally important QTLs have been originally described and quantified in our previous study (Sa et al. 2015). We previously assessed ten grain yield traits at the Maize Experiment Station including: ST, PH, EH, ear length (EL), ear diameter (ED), setted ear length (SEL), fresh 100 kernel weight $(100 \mathrm{KW})$, total kernel weight (TKW), water content (WC) and amylose content (AC). Inclusive composite interval mapping was performed to identify QTLs using the ICIM-ADD module of QTL IciMapping, version 4.0 (Li et al. 2007). The scanning step for all traits was set to $1 \mathrm{cM}$. Reference LOD values were obtained after 1000 permutations with the Type I error (false positives) set to 0.05 . The LOD threshold to affirm the presence of a QTL was set to 3.0. 


\section{Results}

\section{Mu-TD and Ti-derived SCAR profile}

Here, we analyzed DNA polymorphism occurring between the two parents, Mo17 and KW7, using $M u$-TD and Ti-SCAR analysis. $M u$-TD generated multiple bands for each primer combination that resulted in a highly polymorphic genetic profile (Table 2). The overall amplification profiles were determined to be rather similar to those observed with conventional AFLP. A total of 635 fragments from 20 primer combinations were scored, and the number of fragments ranged from $15(M u \mathrm{G} 1-8)$ to $48(\mathrm{MuG2}-2)$. Among $635 \mathrm{Mu}$-TD fragments, 380 (60\%) were determined to be polymorphic between the parental lines which were successfully segregating in RILs. At a 5\% significance level, we observed $55 \mathrm{Mu}$-TD markers (23 in Mo17 and 32 in KW7), which deviated from Mendelian segregation (an assumed 1:1 ratio). Additionally the polymorphic fragments with frequency $>20 \%$ were eliminated from further analysis. Polymorphisms in the presence or absence of a parental amplicon were found in all 34 Ti-SCAR primer combinations.

\section{Linkage map construction}

In total, 1,029 markers were analyzed in the RIL population, and 907 (259 Mu-TD, 34 Ti-SCARs, 608 SSRs and 6 SNP) were placed on the linkage map (Table 3, Fig. 1). On this linkage map, 24 linkage groups were formed, 10 of which were assigned to a chromosome. All unassigned linkage groups (total $=122$ or $34 \%$ ) contained four or fewer markers and were excluded from further analysis. The length of the genetic map spanned $6,248.2 \mathrm{cM}$ across all

Table 2. Polymorphism detected in the Mo17 $\times$ KW7 RIL mapping population

\begin{tabular}{lcccc}
\hline \hline & \multicolumn{2}{c}{ Number of amplified fragments } & & \\
\cline { 2 - 3 } Mu primers & $\begin{array}{c}\text { Monomorphic } \\
\text { bands }\end{array}$ & $\begin{array}{c}\text { Polymorphic } \\
\text { bands }\end{array}$ & Total & $\begin{array}{c}\% \\
\text { Polymorphic }\end{array}$ \\
\hline$M u \mathrm{G} 1-1$ & 15 & 27 & 42 & 64 \\
$M u \mathrm{G} 1-3$ & 12 & 32 & 44 & 73 \\
$M u \mathrm{G} 1-4$ & 20 & 14 & 34 & 41 \\
$M u \mathrm{G} 1-8$ & 9 & 6 & 15 & 40 \\
$M u \mathrm{G} 2-2$ & 14 & 34 & 48 & 71 \\
$M u \mathrm{G}-4$ & 10 & 17 & 27 & 63 \\
$M u \mathrm{G} 3-2$ & 15 & 32 & 47 & 68 \\
$M u \mathrm{G} 3-4$ & 17 & 28 & 45 & 62 \\
$M u \mathrm{G} 4-1$ & 18 & 13 & 31 & 42 \\
$M u \mathrm{G} 4-2$ & 17 & 9 & 26 & 35 \\
$M u \mathrm{G} 4-3$ & 12 & 20 & 32 & 63 \\
$M u \mathrm{G} 4-4$ & 12 & 19 & 31 & 61 \\
$M u \mathrm{G} 4-6$ & 12 & 18 & 30 & 60 \\
$M u \mathrm{G} 5-1$ & 14 & 12 & 26 & 46 \\
$M u \mathrm{G} 5-2$ & 6 & 16 & 22 & 73 \\
$M u \mathrm{G} 6-2$ & 12 & 5 & 17 & 29 \\
$M u \mathrm{G} 7-1$ & 8 & 30 & 38 & 79 \\
$M u \mathrm{G} 7-3$ & 12 & 17 & 29 & 59 \\
$M u \mathrm{G} 7-4$ & 10 & 20 & 30 & 67 \\
$M u \mathrm{G} 7-6$ & 10 & 11 & 21 & 52 \\
$\mathrm{Total}$ & 255 & 380 & 635 & 60 \\
\hline
\end{tabular}

Table 3. Distribution of $M U$-TD, SCARs, SSRs and SNP markers across maize chromosomes, and the chromosome length $(\mathrm{cM})$ in the RIL mapping population

\begin{tabular}{|c|c|c|c|c|c|c|c|}
\hline \multirow{2}{*}{$\begin{array}{l}\text { Chromo- } \\
\text { some }\end{array}$} & \multicolumn{4}{|c|}{ Number of markers } & \multirow{2}{*}{$\begin{array}{c}\text { Total } \\
\text { markers }\end{array}$} & \multirow{2}{*}{$\begin{array}{l}\text { Length } \\
(\mathrm{cM})\end{array}$} & \multirow{2}{*}{$\begin{array}{c}\text { Avg. loci } \\
\text { interval }\end{array}$} \\
\hline & $M u$-TD & SCAR & SSR & SNP & & & \\
\hline 1 & 32 & 5 & 62 & & 99 & 789.8 & 7.98 \\
\hline 2 & 27 & 4 & 59 & & 90 & 698.6 & 7.76 \\
\hline 3 & 40 & 1 & 59 & 1 & 101 & 862.1 & 8.54 \\
\hline 4 & 45 & 2 & 71 & 2 & 120 & 786.3 & 6.55 \\
\hline 5 & 20 & 4 & 68 & 1 & 93 & 519.1 & 5.58 \\
\hline 6 & 23 & 3 & 54 & & 80 & 543.7 & 6.80 \\
\hline 7 & 11 & 3 & 58 & & 72 & 481.5 & 6.69 \\
\hline 8 & 23 & 1 & 67 & & 91 & 619.3 & 6.81 \\
\hline 9 & 23 & 6 & 62 & 2 & 93 & 547.3 & 5.89 \\
\hline 10 & 15 & 5 & 48 & & 68 & 400.5 & 5.89 \\
\hline Total & 259 & 34 & 608 & 6 & 907 & $6,248.2$ & 6.84 \\
\hline
\end{tabular}

linkage groups, where the average interval between markers was $6.84 \mathrm{cM}$. Additionally, we observed high variation in the number of integrated markers across all ten maize chromosomes. For instance, chromosome four exhibited the highest number of integrated markers (45 Mu-TD and 2 TiSCARs), compared to chromosome seven, which possessed the fewest number of integrated markers ( $11 \mathrm{Mu}$-TD and 3 Ti-SCARs). The length of each chromosome was also highly variable. For instance, the length of each chromosome ranged from $400.5 \mathrm{cM}$ (chromosome 10) to $862.1 \mathrm{cM}$ (chromosome three). In our analysis, most of the $M u$-TD and SCARs were distributed throughout the ten maize chromosomes. Among the six mapped SNP loci, three were linked with $M u$-TD loci: locus sh2 was located on chromosome three and linked to flanking SSR locus umc 2174 and $M u$ TD locus $M u$ G1-1-1 at a distance of $2.78 \mathrm{cM}$ and $13.4 \mathrm{cM}$, respectively. Similarly, sul was located on chromosome four and linked to loci bnlg 265 and $M u$ G4-3-17, separated by 1.34 and $12.6 \mathrm{cM}$, respectively. $W x 1$ was located on chromosome nine and flanked by loci umc1634 and $M u \mathrm{G} 1-$ 4-14, which were separated by $5.09 \mathrm{cM}$ and $11.8 \mathrm{cM}$, respectively.

\section{Quantitative trait loci with additive effect}

Our QTL analysis, performed using the ICIM method, detected 24 significant QTLs associated with nine traits (Table 4, Fig. 2). All eight agriculturally important traits except $\mathrm{PH}$ involved $M u$ loci associated with QTLs, among them, five traits, namely. EL, 100KW, WC, ED, and AMY, were linked exclusively to seven $M u$ loci, whereas two traits (ED and AMY) were associated with SCARs. These are the newly identified QTLs compared to our previous study (Sa et al. 2015). All nine traits detected QTLs were mapped onto each chromosome with the exception of chromosome three. The number of QTLs for each trait ranged from two (ST, PH, EH) to four (EL and SEL); however, we did not observe any QTL associations with TKW. The additive effect for 18 QTLs was positive, with Mo17 increasing the effect of QTLs, whereas eight QTLs were negative, with KW7 increasing the effect. 

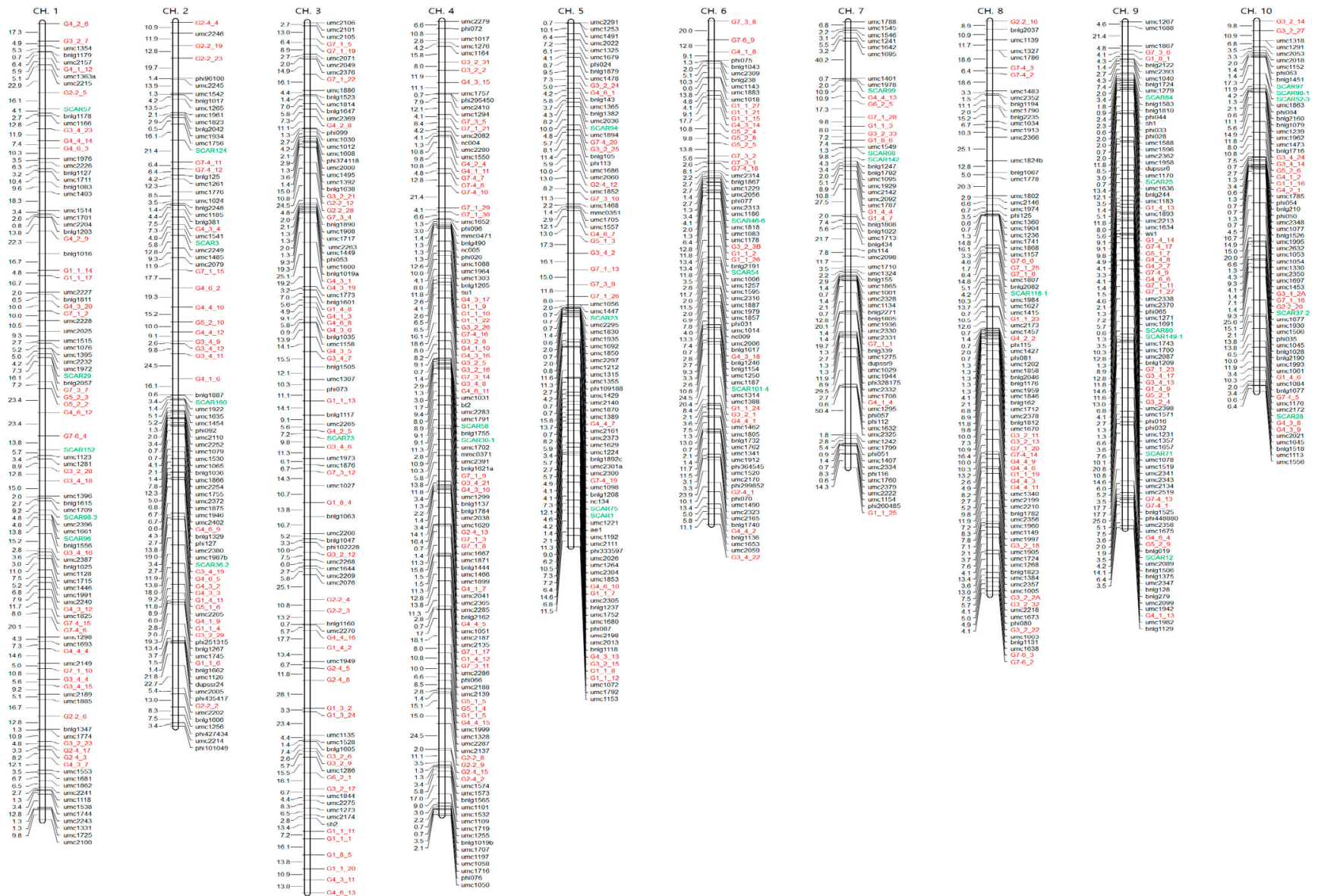

Fig. 1. Genetic linkage map based on $M u$-TD, Ti-derived SCARs, SSRs and SNP markers. A total of $259 M u$-TDs and 34 SCARs were assigned throughout the 10 maize chromosomes using a previously established map of 608 SSRs and 6 SNPs. Map distances (left) are expressed in cM. $M u$-TDs and SCARs are shown in red and green letters, respectively, and SSRs and SNPs are shown in black letters (right).

\section{Discussion}

TEs, mobile components of the genome, are potential sources of genetic variation in agriculturally important crops and can generate important changes at both genetic and phenotypic levels. Several characteristics of TEs make them especially useful for generating molecular markers, including their ability to create unique insertion polymorphisms and their wide distribution throughout the genome. With a variety of molecular techniques, both RNA and DNA transposon superfamilies have been utilized to develop new genetic markers for agriculturally important crops (Kalendar and Schulman 2006, Kwon et al. 2005, Lee et al. 2005, Queen et al. 2004, Yaakov et al. 2012).

This study describes the utility of both $M u$ and CACTA elements derived from SCAR markers for both genetic mapping and identifying QTLs related to agronomic traits. To accomplish this, we used previously developed RIL populations derived from a cross between dent corn (Mo17) and a waxy corn (KW7). Mutator-based transposon display analysis $(M u$-TD) or the RIL populations revealed high levels of $M u$ element polymorphism (60\%). Past research has shown similarly high polymorphism rates $(51 \%)$ obtained from $M u$-TD analysis using SSR markers in the same RIL population (Sa et al. 2012), as well as other marker systems developed for maize such as RFLPs $(50-80 \%)$ (Beavis and Grant 1991, Gardiner et al. 1993), AFLPs (21-47\%) (Castiglioni et al. 1999, Zhang et al. 2006), and MITE-TD (60\%) (Casa et al. 2000). Additionally, segregation analysis revealed $15 \%$ distortion among markers $(\alpha=0.05)$, which is similar to our previous study using SSR markers (14.9\%) (Sa et al. 2012). Segregation distortion, or deviations from Mendelian inheritance, is a common phenomenon observed in other marker systems and is caused by biotic factors such as pollen tube competition, preferential fertilization, sterility, chromosomal rearrangements (e.g., translocation) and, residual heterozygosity (Casa et al. 2000, Lu et al. 2002, Zhu et al. 2007). We also observed non-parental banding patterns (patterns not found in either parental profile) in $0.21 \%$ of the total fragments, which was lower compared to our previous research using SSR markers (3.5\%) (Sa et al. 2012). This phenomenon is commonly observed with transposon-based marker systems used in maize and other agriculturally important crops (Casa et al. 2000, Kwon et al. 
Table 4. Detection of QTLs for agronomic traits in RIL $7: 8$ population based on additive effect

\begin{tabular}{|c|c|c|c|c|c|c|}
\hline Trait name & Chromosome & Left marker & Right marker & 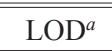 & $\mathrm{PVE}^{b}$ & $\operatorname{Add}^{c}$ \\
\hline ST & 2 & *G2-4-4 & *umc2246 & 3.0725 & 14.2621 & 0.0441 \\
\hline ST & 6 & *phi070 & *umc1490 & 3.561 & 14.8327 & -0.0456 \\
\hline PH & 4 & *umc1667 & *umc1871 & 4.9022 & 19.6202 & 7.5419 \\
\hline $\mathrm{PH}$ & 8 & *umc1139 & *umc1327 & 2.5175 & 9.558 & 5.232 \\
\hline $\mathrm{EH}$ & 4 & *umc1667 & *umc1871 & 2.7144 & 13.2952 & 4.4243 \\
\hline $\mathrm{EH}$ & 6 & *G2-4-1 & *phi070 & 2.5377 & 14.5871 & 4.6504 \\
\hline EL & 6 & $* \mathrm{G} 7-6-9$ & $*^{*} \mathrm{G} 4-1-8$ & 3.1218 & 8.5512 & 0.3498 \\
\hline EL & 6 & *G4-1-8 & *phi075 & 7.0729 & 18.467 & -0.5141 \\
\hline EL & 9 & *G3-2-4 & *umc2398 & 3.3888 & 7.6276 & 0.3305 \\
\hline Fre $100 \mathrm{KW}$ & 7 & *G1-4-4 & $* \mathrm{G} 1-4-7$ & 3.0776 & 14.0599 & -1.2337 \\
\hline Fre $100 \mathrm{KW}$ & 10 & *umc1506 & *phi035 & 4.4863 & 19.1382 & 1.4869 \\
\hline WC & 1 & *umc1744 & *umc 2243 & 3.7039 & 13.895 & 0.3639 \\
\hline WC & 8 & *umc2199 & *umc2210 & 2.7817 & 10.6216 & 0.3158 \\
\hline WC & 9 & *G3-4-13 & *G1-4-9 & 4.4257 & 16.8985 & -0.3995 \\
\hline SEL & 6 & $* \mathrm{G} 7-6-9$ & *G4-1-8 & 2.7277 & 7.6262 & 0.3839 \\
\hline SEL & 6 & *G4-1-8 & *phi075 & 5.383 & 14.8862 & -0.5364 \\
\hline SEL & 10 & *G3-2-27 & *umc1318 & 2.5362 & 6.7813 & 0.3624 \\
\hline ED & 2 & *umc2214 & *phi101049 & 2.7322 & 6.1104 & 0.0667 \\
\hline ED & 7 & SCAR68 & SCAR142 & 4.8524 & 12.0571 & -0.4194 \\
\hline ED & 10 & *G3-4-2 & $* \mathrm{G} 7-1-16$ & 3.8628 & 8.6832 & 0.0792 \\
\hline AMY & 5 & *G4-6-10 & *G1-1-7 & 3.8043 & 1.818 & -0.6296 \\
\hline AMY & 7 & SCAR68 & SCAR142 & 4.4493 & 2.433 & -3.2419 \\
\hline AMY & 9 & *umc1634 & $*_{\mathrm{wx} 1}$ & 47.7522 & 84.8132 & 4.3163 \\
\hline AMY & 9 & $* \mathrm{G} 7-1-23$ & *G3-4-17 & 4.5097 & 2.2172 & 0.6963 \\
\hline
\end{tabular}

${ }^{a}$ Logarithm of the odds ratio.

${ }^{b}$ Percent of phenotypic variation explained (\%).

${ }^{c}$ Additive effect, where negative value represents QTLs effect contributed by KW7 and positive value represent QTLs effect contributed by Mo17.

Chr. 1

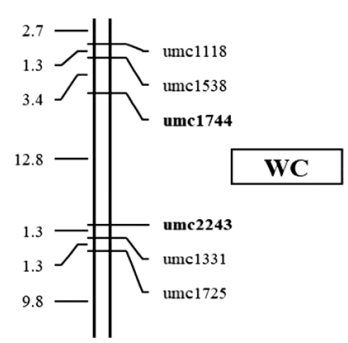

Chr. 2

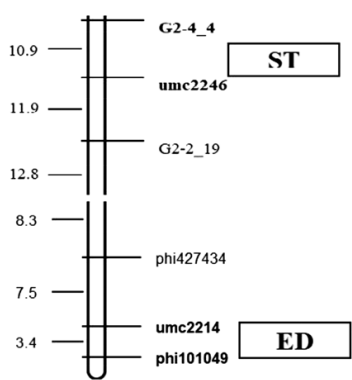

Chr. 4

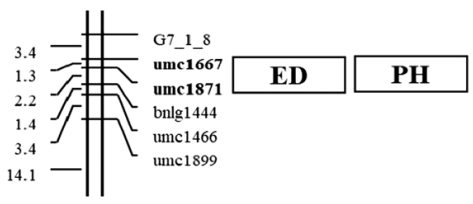

Chr. 5

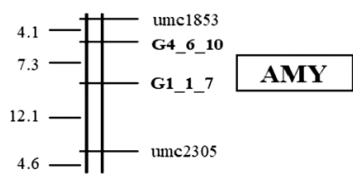

Chr. 6

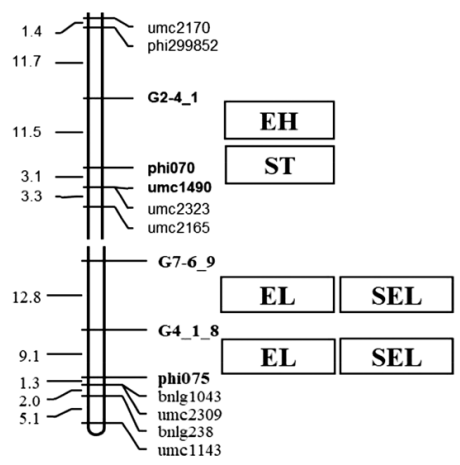

Chr. 7

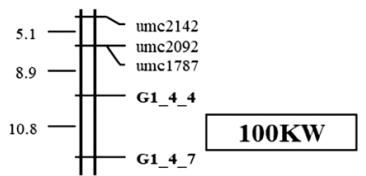

1.3 - umc1549

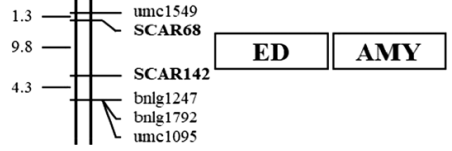

Chr. 8
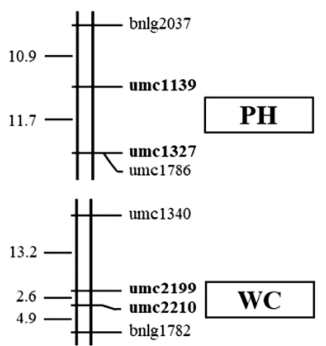

Chr. 9

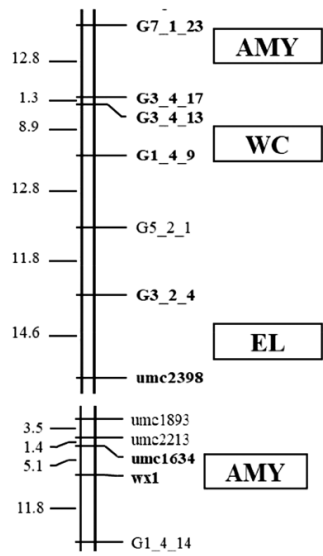

Chr. 10

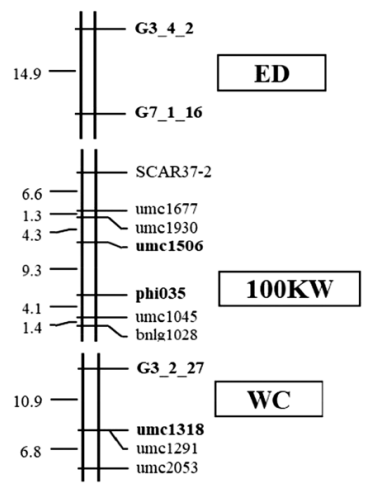

Fig. 2. Chromosomal location of quantitative trait loci (QTL). Map distance (on left) are given in cM (Kosambi function). Markers in bold are flanking markers for QTLs represented by respective traits (quadrangle on right). 
2006, Lee et al. 2006). This widely observed result may be driven by factors such as: inbreeding depression, a mutation in a restriction site, pollen contamination, residual heterozygosity, or genomic rearrangements (Casa et al. 2000, Ramekar et al. 2015).

Our use of $258 \mathrm{Mu}$-TD and 34 SCAR markers resulted in $6,248.2 \mathrm{cM}$ of additional length to the existing linkage map. However, we eliminated a high number of markers $(34 \%)$ in the linkage group, possibly due to low replication of RILs $(\mathrm{N}=80)$. Additionally, chromosomal distribution of transposon-based markers may differ between plant species and between transposon families. For example, in rice, Rim 2/ Hipa CACTA transposons were distributed in pericentric regions (Lee et al. 2006), whereas in maize, the Heartbreaker MITE loci and Isacc-TD loci were distributed throughout the chromosomes (Casa et al. 2000, Lee et al. 2005). Here, we found $M u$-TD loci to be present on both arms of each chromosome and distributed throughout the ten chromosomes. For several chromosomes, $M u$-TD loci tended to cluster on the linkage map. The clustering of markers is not uncommon. For example, our approach using TD is similar to using AFLPs, and previous studies have shown clustering of AFLP markers on linkage maps (Becker et al. 1995, Castiglioni et al. 1999, Okuda et al. 2012). This may occur in AFLP analyses because adenine and thymine rich regions are digested by restriction enzymes or because chromosomal recombination is repressed in the centromeric or telomeric regions (Stich et al. 2006, Van Eck et al. 1995, Wang et al. 2004). Moreover, Manninen et al. (2000) and Rodriguez et al. (2006) observed similar results using retrotransposonbased markers in barley and suggested that marker clustering may be related to high copy number or the nested insertion of retrotransposons. In our analysis, CACTA-derived SCAR markers were found to integrate between SSR markers, which resulted in minor variations in map length. Nevertheless, here we demonstrate that transposon-derived SCAR markers are capable of identifying polymorphisms in RIL populations, and can be a simple, time-saving alternate to marker development. In our previous study (Sa et al. 2015), we used six sets of SNP primers to determine the chromosomal location of genes involved in starch synthesis. To accomplish this, we used the same SNPs as anchor markers and uncovered three SNPs linked to $M u$-TD loci. Future studies would benefit from investigating the physical linkage between starch synthesis genes and DNA transposons, which would help build a more detailed genetic map and provide new insights into gene regulation of agriculturally important traits. Moreover, our future work will focus on designing Mutator-derived SCAR markers in maize.

Based on the integrated linkage map reported here, we have detected 24 QTLs linked to nine traits located on maize chromosomes. Among these, 6 QTLs were identified using $M u$ loci co-localized with SSR markers whereas 9 QTLs were located on chromosome 5, 6, 7, 9 and 10 involved only $M u$ loci or SCARs. These QTLs, involving DNA transposon-based markers for traits AMY, 100KW,
WC, SEL, EL, and ED are newly found compared to our previous study (Sa et al. 2015) and are valuable sources. The traits investigated here are agronomically important and describing QTL flanking molecular markers is a vital contribution to future breeding programs. Note that because we used one year of phenotyping data, we cannot account for environmental effects on the QTLs. Nonetheless, this study successfully located QTLs linked to $M u$ loci and Ti-derived SCAR markers and is a valuable contribution to ongoing maize breeding programs. Our future work will use TE-based approaches to identify agriculturally important QTLs in response to environmental variations.

\section{Acknowledgments}

This study was supported by the Cooperative Research Program for Agriculture Science \& Technology Development (Project title \#PJ01315701, Project \#PJ013157) Rural Development Administration, Republic of Korea.

\section{Literature Cited}

Beavis, W.D. and D. Grant (1991) A linkage map based on information from four $\mathrm{F}_{2}$ populations of maize (Zea mays L.). Theor. Appl. Genet. 82: 636-644.

Becker, J., P. Vos, M.Kuiper, F. Salamini and M.Heun (1995) Combined mapping of AFLP and RFLP markers in barley. Mol. Gen. Genet. 249: 65-73.

Bennetzen, J.L. (1996) The Mutator transposable element system of maize. Curr. Top. Microbiol. Immunol. 204: 195-229.

Bernardo, R. (2008) Molecular markers and selection for complex traits in plants: learning from the last 20 years. Crop Sci. 48: 1649-1664.

Branco, C.J., E.A.Vieira, G. Malone, M.M.Kopp, E. Malone, A. Bernardes, C.C.Mistura, F.I.Carvalho and C.A. Oliveira (2007) IRAP and REMAP assessments of genetic similarity in rice. J. Appl. Genet. 48: 107-113.

Brunner, S., K. Fengler, M. Morgante, S. Tingey and A. Rafalski (2005) Evolution of DNA sequence nonhomologies among maize inbreds. Plant Cell 17: 343-360.

Butelli,E., C.Licciardello, Y.Zhang, J.Liu, S. Mackay, P.Bailey, G. Reforgiato-Recupero and C.Martin (2012) Retrotransposons control fruit-specific, cold-dependent accumulation of anthocyanins in blood oranges. Plant Cell 24: 1242-1255.

Casa,A.M., C. Brouwer, A. Nagel, L.Wang, Q.Zhang, S. Kresovich and S.R.Wessler (2000) The MITE family heartbreaker $(\mathrm{Hbr})$ : molecular markers in maize. Proc. Natl. Acad. Sci. USA 97: 10083-10089.

Castiglioni,P., P.Ajmone-Marsan, R. Van Wijk and M.Motto (1999) AFLP markers in a molecular linkage map of maize: codominant scoring and linkage group ditsribution. Theor. Appl. Genet. 99: 425-431.

Collard, B.C.Y., M.Z.Z. Jahufer, J.B. Brouwer and E.C.K. Pang (2005) An introduction to markers, quantitative trait loci (QTL) mapping and marker-assisted selection for crop improvement: The basic concepts. Euphytica 142: 169-196.

Diao, X.M. and D.Lisch (2006) Mutator transposon in maize and MULEs in the plant genome. Acta Genet. Sin. 33: 477-487.

Du, C., N.Fefelova, J.Caronna, L.He and H.K. Dooner (2009) The 
polychromatic Helitron landscape of the maize genome. Proc. Natl. Acad. Sci. USA 106: 19916-19921.

Fan, L., L. Quan, X. Leng, X. Guo, W.Hu, S. Ruan, H. Ma and M.Zeng (2008) Molecular evidence for post-domestication selection in the Waxy gene of Chinese waxy maize. Mol. Breed. 22: 329-338.

Gardiner, J.M., E.H.Coe, S. Melia-Hancock, D.A.Hoisington and S. Chao (1993) Development of a core RFLP map in maize using an immortalized $F_{2}$ population. Genetics 134: 917-930.

Gaut,B.S. (2002) Evolutionary dynamics of grass genomes. New Phytol. 154: 15-28.

Grover,A. and P.C. Sharma (2016) Development and use of molecular markers: past and present. Crit. Rev. Biotech. 36: 290-302.

Gupta,P.K., S. Rustgi and N.Kumar (2006) Genetic and molecular basis of grain size and grain number and its relevance to grain productivity in higher plants. Genome 49: 565-571.

Kalendar, R. and A.H. Schulman (2006) IRAP and REMAP for retrotransposon-based genotyping and fingerprinting. Nat. Protoc. 1: 2478-2484.

Ketthaisong, D., B. Suriharn, R. Tangwongchai and K. Lertrat (2013) Changes in physicochemical properties of waxy corn starches at different stages of harvesting. Carbohydr. Polym. 98: 241-248.

Klosgen, R.B., A. Gierl, Z. Schwarz-Sommer and H.Saedler (1986) Molecular analysis of the waxy locus of Zea mays. Mol. Genet. Genomics 203: 237-244.

Kobayashi, S., N. Goto-Yamamoto and H.Hirochika (2004) Retrotransposon-induced mutations in grape skin color. Science 304: 982

Korswagen,H.C., R.M. Durbin, M.T. Smits and R.H.A. Plasterk (1996) Transposon Tc1-derived, sequence-tagged sites in Caenorhabditis elegans as markers for gene mapping. Proc. Natl. Acad. Sci. USA 93: $14680-14685$.

Kosambi,D.D. (1943) The estimation of map distances from recombination values. Ann. Eugen. 12: 172-175.

Kwon, S.J., K.C.Park, J.H. Kim, J.K. Lee and N.S. Kim (2005) Rim 2/ Hipa CACTA transposon display: a new genetic marker technique in Oryza species. BMC Genet. 6: 15.

Kwon, S.J., S.W.Hong, J.H.Son, J.K.Lee, Y.S.Cha, M.Y.Eun and N.S.Kim (2006) CACTA and MITE transposon distributions on a genetic map of rice using $\mathrm{F}_{15}$ RILs derived from Milyang 23 and Gihobyeo hybrids. Mol. Cells 21: 360-366.

Lee, J.K., S.J.Kwon, K.C.Park and N.S.Kim (2005) Isaac-CACTA transposons: new genetic markers in maize and sorghum. Genome 48: 455-460.

Lee, J.K., J.Y.Park, J.H.Kim, S.J.Kwon, J.H. Shin, S.K.Hong, H.K. Min and N.S.Kim (2006) Genetic mapping of the Isaac-CACTA transposon in maize. Theor. Appl. Genet. 113: 16-22.

Li, H., G. Ye and J. Wang (2007) A modified algorithm for the improvement of composite interval mapping. Genetics 175: 361-374.

Lisch, D. (2002) Mutator transposons. Trends Plant Sci. 7: 498-504.

Lisch, D. (2013) How important are transposons for plant evolution? Nat. Rev. Genet. 14: 49-61.

Lu,H., J.Romero-Severson and R.Bernardo (2002) Chromosomal regions associated with segregation distortion in maize. Theor. Appl. Genet. 105: 622-628.

Manninen, O., R. Kalendar, J. Robinson and A.H.Schulman (2000) Application of $B A R E-1$ retrotransposon markers to the mapping of a major resistance gene for net blotch in barley. Mol. Gen. Genet. 264: 325-334.

Meng, L., H.Li, L.Zhang and J.Wang (2015) QTL IciMapping: Integrated software for genetic linkage map construction and quantitative trait locus mapping in biparental populations. Crop J. 3: 269-
283.

Morgante, M., E. De Paoli and S.Radovic (2007) Transposable elements and the plant pan-genomes. Curr. Opin. Plant Biol. 10: 149155.

Nelson, O.E. and H.W. Rines (1962) The enzymatic deficiency in the waxy mutant of maize. Biochem. Biophys. Res. Commun. 9: 297300.

Okuda,Y., J.Ueda, Y.Obatake, S.Murakami, Y.Fukumasa and T. Matsumoto (2012) Construction of a genetic linkage map based on amplified fragment length polymorphism markers and development of sequence-tagged site markers for marker-assisted selection of the sporeless trait in the oyster mushroom (Pleurotus eryngii). Appl. Environ. Microbiol. 78: 1496-1504.

Park, K.J., K.J. Sa, H.-J. Koh and J.K. Lee (2013) QTL analysis for eating quality-related traits in an $\mathrm{F}_{2: 3}$ population derived from waxy corn $\times$ sweet corn cross. Breed. Sci. 63: 325-332.

Peng, B., Y.Li, Y.Wang, C.Liu, Z.Liu, W.Tan, Y.Zhang, D.Wang, Y. Shi, B. Sun et al. (2011) QTL analysis for yield components and kernel-related traits in maize across multi-environments. Theor. Appl. Genet. 122: 1305-1320.

Prasanna, B.M., K. Pixley, M.L. Warburton and C.-X.Xie (2010) Molecular marker-assisted breeding options for maize improvement in Asia. Mol. Breed. 26: 339-356.

Queen, R.A., B.M. Gribbon, C. James, P. Jack and A.J.Flavell (2004) Retrotransposon-based molecular markers for linkage and genetic diversity analysis in wheat. Mol. Genet. Genom. 271: 91-97.

Ramekar,R.V., K.J.Sa, S.Y.Woo and J.K.Lee (2015) Non-parental banding patterns in recombinant inbred line population of maize with SSR markers. Genet. Mol. Res. 14: 8420-8430.

Ramekar, R.V., K.-C.Park, K.J.Sa and J.K.Lee (2018) Mutator-based transposon display: a genetic tool for evolutionary and crop improvement studies in maize. Mol. Biotechnol. doi: 10.1007/s12033018-0118-z.

Robertson, D.S. (1978) Characterization of a mutator system in maize. Mutat. Res. 51: 21-28.

Rodriguez,M., D.O'sullivan, P.Donini, R.Papa, E.Chiapparino, F. Leigh and G.Attene (2006) Integration of retrotransposons-based markers in a linkage map of barley. Mol. Breed. 17: 173-184.

Roy, N.S., K.C.Park, S.I.Lee, M.J.Im, R.V.Ramekar and N.S.Kim (2017) Development of CACTA transposon derived SCAR markers and their use in population structure analysis in Zea mays. Genetica 146: 1-12.

Sa, K.J., J.Y.Park, K.-C.Park and J.K. Lee (2012) Analysis of genetic mapping in a waxy/dent maize RIL population using SSR and SNP markers. Genes Genom. 34: 157-164.

Sa, K.J., J.Y.Park, S.Y.Woo, R.V.Ramekar, C.S. Jang and J.K. Lee (2015) Mapping of QTL traits in corn using a RIL population derived from a cross of dent corn $\times$ waxy corn. Genes Genomics 37: $1-14$.

Salvi, S., G. Sponza, M. Morgante, D. Tomes, X. Niu, K.A. Fengler, R. Meeley, E.V.Ananiev, S. Svitashev, E. Bruggemann et al. (2007) Conserved noncoding genomic sequences associated with a flowering-time quantitative trait locus in maize. Proc. Natl. Acad. Sci. USA 104: 11376-11381.

Schnable, P.S., D. Ware, R.S.Fulton, J.C.Stein, F. Wei, S. Pasternak, C.Liang, J.Zhang, L.Fulton, T.A.Graves et al. (2009) The B73 maize genome: complexity, diversity, and dynamics. Science 326: $1112-1115$.

Semagn, K., Å. Bjørnstad and M.N.Ndjiondjop (2006) Principles, requirements and prospects of genetic mapping in plants. Afr. J. Biotechnol. 5: 2569-2587. 
Springer, N.M. and R.M. Stupar (2007) Allelic variation and heterosis in maize: how do two halves make more than a whole? Genome Res. 17: 264-275.

Stich,B., H.P. Maurer, A.E. Melchinger, M.Frisch, M.Heckenberger, J.R. Van Der Voort, J.Peleman, A.P. Sørensen and J.C. Reif (2006) Comparison of linkage disequilibrium in elite European maize inbred lines using AFLP and SSR markers. Mol. Breed. 17: 217-226.

Studer,A., Q.Zhao, J. Ross-Ibarra and J. Doebley (2011) Identification of a functional transposon insertion in the maize domestication gene $t b 1$. Nat. Genet. 43: 1160-1163.

Van Eck, H.J., J.R. Van Der Voort, J. Draaistra, P. Van Zandvoort, E. Van Enckevort, B. Segers, J. Peleman, E. Jacobsen, J. Helder and J. Bakker (1995) The inheritance and chromosomal localization of AFLP markers in a non-inbred potato offspring. Mol. Breed. 1: 397-410.

Vitte, C., T.Ishii, F.Lamy, D. Brar and O.Panaud (2004) Genomic paleontology provides evidence for two distinct origins of Asian rice (Oryza sativa L.). Mol. Genet. Genomics 272: 504-511.

Wang, S., Z. Bao, J.Pan, L.Zhang, B. Yao, A.Zhan, K. Bi and Q.Zhang (2004) AFLP linkage map of an intraspecific cross in Chlamys farreri. J. Shellfish Res. 23: 491-500.

Wang, Y., M.Xu, D.Deng and Y.Bian (2008) Maize Mutator transposon. Front. Agric. China 2: 396-403.

Wicker, T., F. Sabot, A. Hua-Van, J.L. Bennetzen, P. Capy, B. Chalhoub, A. Flavell, P. Leroy, M. Morgante, O. Panaud et al. (2007) A unified classification system for eukaryotic transposable elements. Nat. Rev. Genet. 8: 973-982.

Xu,Y., D.J.Skinner, H.Wu， N.Palacios-Rojas，J.L.Araus，J.Yan, S. Gao, M.L. Warburton and J.H.Crouch (2009) Advances in maize genomics and their value for enhancing genetic gains from breeding. Int. J. Plant Genomics 2009: 957602.

Yaakov, B., E. Ceylan, K. Domb and K. Kashkush (2012) Marker utility of miniature inverted-repeat transposable elements for wheat biodiversity and evolution. Theor. Appl. Genet. 124: 1365-1373.

Yang, G., Y.Li, Q.Wang, Y.Zhou, Q.Zhou, B.Shen, F.Zhang and X. Liang (2011) Detection and integration of quantitative trait loci for grain yield components and oil content in two connected recombinant inbred line populations of high-oil maize. Mol. Breed. 29: 313-333.

Zhang, F., X.Q. Wan and G.T.Pan (2006) QTL mapping of Fusarium moniliforme ear rot resistance in maize. 1. Map construction with microsatellite and AFLP markers. J. Appl. Genet. 47: 9-15.

Zhang, Y., Y.Li, Y.Wang, B.Peng, C.Liu, Z.Liu, W.Tan, D. Wang, Y.Shi, B.Sun et al. (2011) Correlations and QTL detection in maize family per se and testcross progenies for plant height and ear height. Plant Breed. 130: 617-624.

Zhang,Z., Z.Liu, Z.Cui, Y.Hu, B.Wang and J.Tang (2013) Genetic analysis of grain filling rate using conditional QTL mapping in maize. PLoS ONE 8: e56344.

Zhong, W.J., M.D.Zhang, L.Q.Yang, M.C.Wang, Y.L.Zheng, W.P. Yang and Y.J.Gao (2012) Isolating the mutator transposable element insertional mutant gene miol6 of maize using double selected amplification of insertion flanking fragments (DSAIFF). J. Integr. Agric. 11: 1592-1600.

Zhu, C., C. Wang and Y.M.Zhang (2007) Modeling segregation distortion for viability selection. I. Reconstruction of linkage maps with distorted markers. Theor. Appl. Genet. 114: 295-305. 